\title{
Analysis of DVB-H Network Coverage With the Application of Transmit Diversity
}

\author{
Yue Zhang, C. Zhang, J. Cosmas, K. K. Loo, T. Owens, R. D. Di Bari, Y. Lostanlen, and M. Bard
}

\begin{abstract}
This paper investigates the effects of the Cyclic Delay Diversity (CDD) transmit diversity scheme on DVB-H networks. Transmit diversity improves reception and Quality of Service $(\mathrm{QoS})$ in areas of poor coverage such as sparsely populated or obscured locations. The technique not only provides robust reception in mobile environments thus improving QoS, but it also reduces network costs in terms of the transmit power, number of infrastructure elements, antenna height and the frequency reuse factor over indoor and outdoor environments. In this paper, the benefit and effectiveness of CDD transmit diversity is tackled through simulation results for comparison in several scenarios of coverage in DVB-H networks. The channel model used in the simulations is based on COST207 and a basic radio planning technique is used to illustrate the main principles developed in this paper. The work reported in this paper was supported by the European Commission IST project-PLUTO (Physical Layer DVB Transmission Optimization).
\end{abstract}

Index Terms-CDD, CNR, DVB-T/H, OFDM, QoS, SFN, transmit diversity.

\section{INTRODUCTION}

$\mathbf{T}$ O PROVIDE television services to mobile users, several mobile TV standards including DVB-H (Digital Video Broadcasting-Handheld), T-DMB (Terrestrial Digital Multimedia Broadcasting), 3G-MBMS (3G-Multimedia Broadcast Multicast Service), DMB-H (Digital Multimedia Broadcasting Handheld), MediaFLO and ATSC (Advanced Television Systems Committee) have been proposed by different regions such as Europe, Japan, Korea, China and North America. As for Europe, all TV networks and their corresponding transmitter sites are slowly being replaced by DVB-T/H [1] networks. In coexistence of DVB-T network, the DVB-H mobile services network is regarded as the solution for the provision of localized

Manuscript received September 30, 2007; revised April 14, 2008. Published August 20, 2008 (projected). The work is supported by the European Commission IST project-PLUTO (Physical Layer DVB Transmission Optimization).

Y. Zhang is with the Anritsu Company, Stevenage SG1 2EF, U.K. (e-mail: Yue_Zhang@ieee.org).

C.H. Zhang is with the Ericsson Communications Company Ltd., Beijing 100102, China (e-mail: chunhui.zhang@ericsson.com).

J. Cosmas, K.K. Loo, T. Owens, and R.D. Bari are with the School of Engineering and Design, Brunel University, London UB8 3PH, U.K. (e-mail: John.Cosmas@brunel.ac.uk; Jonathan.Loo@brunel.ac.uk; Thomas.Owens@brunel.ac.uk; Raffaele.DiBari@brunel.ac.uk).

Y. Lostanlen is with the Siradel S.A., Rennes Cedex F-35043, France (e-mail: ylostanlen@siradel.com).

M. Bard is with the Broadreach Communications Ltd., London SW6 6BA,

U.K. (e-mail: mail@broadreachsystems.com).

Color versions of one or more of the figures in this paper are available online at http://ieeexplore.ieee.org.

Digital Object Identifier 10.1109/TBC.2008.2002165

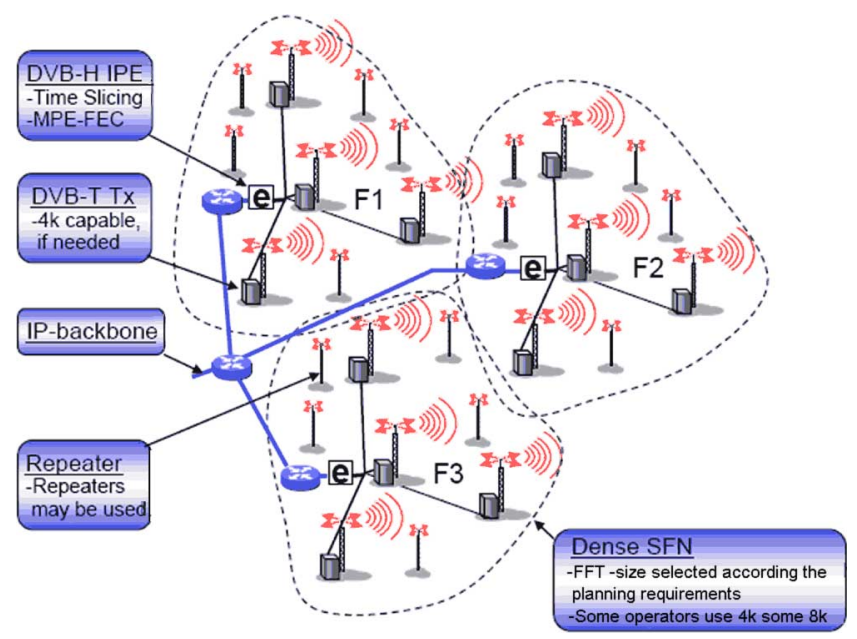

Fig. 1. DVB-H networks.

services as illustrated in Fig. 1. These systems will be deployed mainly in UHF (Ultra High Frequency) and VHF (Very High Frequency) frequency bands.

There are three issues that should be considered before deploying a DVB-H network. First, a low transmit power and antenna height should be used for localized coverage. Second, the frequency reuse pattern for a single frequency network (SFN) [2], [3] should be considered. A SFN can serve an arbitrary large area with the same information broadcasted at the same frequency, resulting in the potential for diversity gain, equivalent to the macro diversity in radio communications. Third, the receivers are mostly in mobile profile and located at street level, experiencing thus mainly non line of sight (NLOS) reception conditions.

Mobile TV systems such as DVB-H are expected to provide mobile services to as wide a coverage area as possible [4]. Therefore, multiple antennas with some form of transmit diversity scheme, i.e. CDD (Cyclic Delay Diversity) which inherently exploits the multipath scattering effect of the wireless channel, are proposed to improve the statistics of the DVB-H [5] receive carrier-to-noise ratio (CNR). This would allow the system to provide wider mobile reception in poor coverage areas such as indoors, sparsely populated and obscured locations. CDD [6]-[8] is a simple and elegant method which when combined with the MIMO (Multi-Input-Multi-Output) technique improves frequency selectivity. The computational cost of CDD is very low as the signal processing it needs is performed on OFDM (Orthogonal Frequency Division Multiplexing) signals in the time domain. For standardized DVB systems, CDD can be implemented provided the modifications 
made to accommodate it keep the systems standards compatible. CDD diversity techniques lower the CNR threshold required by the receiver to achieve a satisfactory quality of service (QoS) and maintain coverage area. This can result in a significant improvement in system performance by a digital TV network [9]-[11] particularly for non line of sight (NLOS) signals at mobile receivers.

This paper investigates through simulations the effect of the CDD transmit diversity scheme on DVB-H networks in terms of coverage, transmit power and frequency reuse pattern. The coverage improvement is with respect to DVB-H networks without the diversity technique. The simulations focus on the diversity gain of CDD over indoor and outdoor channels. It is shown that the diversity scheme not only provides robust reception and QoS in mobile environments but also reduces network costs in terms of the transmit power, number of infrastructure elements, antenna height and the frequency reuse factor. However, because the simulations of this paper investigate reception at the physical layer, mobile reception correction factors such as Channel Estimation, inner Reed-Solomon coding and MPE-FEC (Multiprotocol Encapsulation-Forward Error Correction) have not been modeled.

The rest of the paper is organized as follows Section II discusses the network coverage planning for DVB-H systems. It analyzes the relationship between CNR, transmit power and a basic computation of network coverage. Section III discusses the CDD diversity scheme. Section IV shows how the CDD diversity scheme can be applied to DVB-H cellular networks and provides simulation results showing network coverage improvements in terms of transmitter antenna height and CNR threshold. Finally, Section V discusses the simulations and draws conclusions from them.

\section{Network Coverage PlanNing FOR DVB-H Systems}

In this section, the parameters of DVB-H network coverage planning are derived and discussed under some common accepted assumptions concerning the radio channel. The aim of network planning is to optimize transmitter parameters such as transmitter power and antenna height such that the calculated CNR is above the minimum allowable threshold value. The network coverage is based on the outage probability of the network. Transmit diversity reduces the threshold CNR of DVB-H transmissions thus helping with the network planning.

\section{A. CNR Threshold for DVB-H Network Planning}

In evaluating the performance of DVB-H systems, the CNR threshold at which the receiver can get a predefined QoS is the key parameter affecting the coverage of the network. At the receiver, this threshold characterizes the ability of the receiver to demodulate the signal under different channel profiles and this ability mainly depends on the receiver design. Imperfect symbol and frequency synchronization together with fading and phase noise can increase the CNR threshold for a predefined QoS. Different design algorithms for synchronization, channel estimation, etc., in the receiver give different CNR requirements for the same channel profile.

At an arbitrary receiving location, the received power is affected by the fast fading effects caused by the local multipath propagation and by slow fading due to shadowing. The received $\mathrm{CW}$ (Continuous Wave) signal can be expressed as [12]:

$$
Y(t)=L(t) R(t) e^{j \omega_{c} t+j \Phi(t)}
$$

where $\omega_{c}$ is the angular carrier frequency and, $R(t) e^{j \Phi(t)}$ is a complex Gaussian random variable; The envelope $R(t)$ is Rayleigh distributed and is normalized to

$$
E\left\{R^{2}(t)\right\}=1
$$

while $\Phi(t)$ is a uniformly distributed random phase. The quantity $L(t)$ is caused by shadowing and can be modeled as a wellknown lognormal distribution variable as follows:

$$
P=10 \log _{10} L^{2}
$$

where the random variable $P$ is Gaussian with probability density function:

$$
f(p)=\frac{1}{\sqrt{2 \pi \sigma}} \exp \left(-\frac{(p-m)^{2}}{2 \pi \sigma^{2}}\right) .
$$

where $\sigma$ is the $\mathrm{dB}$ spread (standard deviation), which varies between 6 and $13 \mathrm{~dB}$ depending the severity of the shadowing. The mean value $m$ reflects the median attenuation in signal strength in the mobile environment which is calculated according to ITU P-1546 [13].

For an MFN (Multi Frequency Network) configuration and neglecting the thermal noise the received voltage output at the $i$ th antenna of an M-branch diversity receiver is expressed as [12]:

$$
y_{i}=L_{0} R_{0 i} e^{j \Phi_{0 i}} S_{0}+\sum_{k=1}^{K} L_{k} R_{k i} e^{j \Phi_{k i}} S_{k}, \quad i=1, \cdots, M
$$

where $k=1, \ldots, K$ are associated with the signals from the interference transmitters, $k=0$ is associated with the desired signal, $R_{k i}$ is the Rayleigh fading envelope, $\Phi_{k i}$ is the time varying random phase and $S_{k}$ is the $k$ th modulation that is normalized such that $E\left\{S_{k} \cdot S_{k}^{*}\right\}=1$. Since the modulation bandwidth is much larger than the fading rate, the instantaneous signal power is

$$
S_{t}^{\text {instant }}=L_{0}^{2} R_{0 i}^{2}
$$

and the instantaneous interference power is:

$$
I_{t}^{\text {instant }}=\sum_{k=1}^{K} L_{k}^{2} R_{k i}^{2} .
$$

Considering (2), the average signal power for each diversity branch in $\mathrm{dBW}$ scale is:

$$
U=10 \log _{10}\left(E\left\{S_{\text {instant } t}\right\}\right)=10 \log _{10} L_{0}^{2}
$$

Ignoring thermal noise in the presence of the inner interference and outer interference, the average interference power for each diversity branch in $\mathrm{dBW}$ is

$$
I=10 \log _{10} \sum_{k=1}^{K} L_{k}^{2}
$$


For the $i$ th signal component the received signal power, $P_{i}$ may contribute to the useful part of the combined signal or the interfering part or to both parts depending on the relative delay. The ratio between the useful contribution, $U_{i}$ and the interfering contribution, $I_{i}$, of the $i$ th signal component is modeled by the weighting function $w\left(\tau_{i}-\tau_{0}\right)$ where $\tau_{i}$ represents the signal delay relative to the starting point of the receiver detection window $\tau_{0}[14]$.

$$
\begin{aligned}
U_{i} & =w\left(\tau_{i}-\tau_{0}\right) \cdot P_{i} \\
I_{i} & =\left(1-w\left(\tau_{i}-\tau_{0}\right)\right) \cdot P_{i}
\end{aligned}
$$

For the weighting function $w(\Delta \tau)$, the following quadratic form has been suggested in its simplest form [14] and [18]:

$$
w(\Delta \tau)=\left\{\begin{array}{cl}
0 & \text { if } \Delta \tau \leq 0 \\
1 & \Delta \tau \leq T_{g} \\
\left(\frac{T_{u}-\Delta t+T_{g}}{T_{u}}\right)^{2} & \text { if } T_{g}<\Delta \tau<T_{F} \\
0 & \text { if } \Delta \tau \geq T_{F}
\end{array}\right.
$$

where $T_{u}$ and $T_{g}$ denote the time duration of the useful signal and the guard interval time, $T_{F}$ is the inverse of the pass-band in $\mathrm{Hz}$ of the frequency domain interpolation filter in which the constellation equalization and coherent detection are based on the channel estimation process. The interpolation filter is used for the channel estimation based on the scatter pilot tones in OFDM subcarriers [18]. This value cannot exceed $T_{u} / 3$. Therefore, it is assumed that the index set of the transmitters of the studied SFN is represented by $\Omega=\{1, \ldots, N\}$ and the transmitters of other SFNs operating at the same frequency are denoted by $\Psi=\{1, \ldots, \mathrm{M}\} . P_{i}(\mathrm{~W})$ is the received signal power coming from $i$ th transmitter and usually $P_{i}$ can be represented by a lognormal distribution variable with a mean value of $m_{P}$ and a standard deviation of $\sigma \rho$, the mean of $w \cdot P_{i}$ is $m_{P}+10 \cdot \log (w)$ and the standard deviation is $\sigma \rho$. If the background noise power is $N_{0}$, the CNR ratio can be written as:

$$
\begin{aligned}
\Gamma & =\frac{U}{I+N_{0}}=\frac{\sum U_{i}}{\sum_{i \in \Omega} I_{i}+N_{0}} \\
& =\frac{\sum_{i \in} \cdot w\left(\tau_{i}-\tau_{0}\right)}{\sum_{i \in \Omega} P_{i} \cdot\left(1-w\left(\tau_{i}-\tau_{0}\right)\right)+\sum_{i \in \Psi} P_{i}+N_{0}}
\end{aligned}
$$

In (13), the numerator represents the useful signal and the denominator represents the interference signal plus noise. The total useful signal $U$ and the interference signal $I$, ignoring $N_{0}$ in the presence of the inner interference and outer interference, can be represented by lognormal distribution variables with parameters, $m_{u}$ and $\sigma_{u}, m_{I}$ and $\sigma_{I}$, respectively. In this case, the $\mathrm{CNR}$ ratio in $\mathrm{dB}$ has a normal distribution with mean $m_{\Gamma}=m_{u}-m_{I}$ and standard deviation $\sigma_{\Gamma}^{2}=\sigma_{u}^{2}+\sigma_{I}^{2}$, assuming $U$ and $I$ are uncorrelated, which might be a strong assumption in urban areas at street level where lots of multipath propagation takes place. Furthermore, from (13), the transmitter power $P_{i}$ from the different transmitters affects the CNR ratio $\Gamma$. The different CNR ratio determines the different transmit power.

\section{B. Outage Probability in DVB-H Networks}

Based on the CNR ratio, the performance of a SFN DVB-H network is usually measured by the coverage probability, $P_{c}$, which is defined as the probability that the CNR exceeds a system specific protection ratio $\gamma_{0}$ :

$$
P_{c}=\operatorname{Pr}\left(\Gamma>\gamma_{0}\right)=1-P_{o}
$$

where the $P_{o}$ for which the CNR is less than the threshold value is given by

$$
P_{o}=1-\int_{\gamma_{0}}^{\infty} \frac{1}{\sqrt{2 \pi} \sigma_{\Gamma}} \cdot \exp \left(\frac{-\left(\Gamma-m_{\Gamma}\right)^{2}}{2 \sigma_{\Gamma}^{2}}\right) d \Gamma
$$

From (14), the coverage area for DVB-H systems is based on the threshold of CNR for the mobile receiver. If the probability of receiver CNR over the threshold is above the desired level, this receiver is regarded as receiving an acceptable QoS receiver. Therefore, the improvement of the CNR threshold is very important in determining the network coverage. A transmitter diversity scheme such as CDD can be applied at the transmitter antenna to decrease the threshold value for the CNR at receiver. Then the decreased CNR threshold can lead to an improvement in DVB-H network coverage in terms of CNR, transmitter power and transmitter antenna height. The following section will describe the configuration of CDD in the transmitter site.

\section{CDD TRANSMIT DIVERSITY SCHEME}

In this section, the theoretical expression of CDD is presented as general guidelines for the system design of transmit diversity to enhance the quality of the received signals in a DVB-T/H broadcasting network by changing the channel states.

The OFDM symbols with CDD can be generated from the reference signal symbols by applying a transmit antenna specific cyclic time shift $\delta_{c y}$ and subsequent insertion of the cyclic prefix. In this case, the signal is not truly delayed between respective antennas but cyclically shifted and thus, there are no restrictions on the delay times and there is no additional ISI (intersymbol interference). Therefore, a length $K$ sequence modulates $K$ subcarriers of the OFDM symbol.

$$
s(l)=\frac{1}{\sqrt{K}} \sum_{k=0}^{K-1} S(k) \cdot e^{j \frac{2 \pi}{K} k l}, \quad l=0, \ldots, K-1
$$

Now the space-time code schemes for CDD with $N$ transmit antennas will be an $N K$ matrix. The codeword can be represented as:

$$
C=\left(\begin{array}{cccc}
s(0) & s(1) & \ldots & s(K-1) \\
s(K-1) & s(0) & \ldots & S(K-2) \\
\ldots & \ldots & \ldots & \ldots \\
s(K-N+1) & s(K-N+2) & \ldots & s(K-N)
\end{array}\right)_{N \times K}
$$

where the $n$th antenna transmits sequence $s(0), \ldots, s(K-1)$ with shift $n$, as $s(K-n), s(K-n+1), \ldots, s(K-n-1)$. However, in the simulation, the cyclic shift is about $1 \mu \mathrm{s}$. 
The channel response from transmit antenna $n$ to receive antenna $m$ at time index $t$ can be represented as:

$$
h_{i j}^{t}=\sum_{l=0}^{L-1} \alpha_{m, l}(t) \delta\left(\tau-\tau_{l}\right)
$$

where $\delta$ is the delay per subchannel from a transmit to a receive antenna. The multipath delays $\tau l$ are equal for all subchannels $h_{N_{T}}(t, \tau)$ and $\alpha_{m, l}(t)$ is statistically independent fading for all $N_{T}$ antennas and all $L$ paths According to (16), the transmit symbol from antenna $i$ at time $t$ is given by

$$
\begin{aligned}
& s_{i}(t)=s_{i}\left(\left(t-\delta_{i}^{c y}\right) \bmod \mathrm{K}\right), \\
& t=0, \ldots K-1, i=0, \ldots, N-1
\end{aligned}
$$

where $\delta_{i}^{c y}$ is the cyclic shift in the $i$ th transmitter antenna. The system is equivalent to the transmission of sequence $s_{i}$ over a frequency selective channel via a transmit antenna to a receive antenna, $j=1, \ldots, M$, which can be described as:

$$
h_{1, j}^{e, t}(d)=\sum_{i=1}^{N} h_{i, j}^{t}\left(\left(d-\delta^{c y}\right) \bmod \mathrm{K}\right), \quad j=1, \ldots, M
$$

and the channel impulse response can be described as:

$$
h_{1, j}^{e, t}=\left[h_{1, j}^{e, t}(0), \ldots, h_{1, j}^{e, t}(K-1)\right]
$$

In the frequency domain, the equivalent channel transfer function is expressed as:

$$
H_{1, m}^{e}(k)=\sqrt{\frac{S N R}{N}} \sum_{n=1}^{N} e^{-j \frac{2 \pi}{K} k \delta_{n}^{c y}} \cdot H_{n, m}(k)
$$

where $H_{n, m}(k)$ denotes the channel transfer function from the $n$th transmit antenna to the $m$ th receive antenna and $\delta_{n}^{c y}$ stands for the transmit antenna specific cyclic delay $\left(\delta_{1}^{c y}=0\right)$.

\section{RESUlTS AND Discussions}

The simulations are divided into two stages. First, each simulation is based on the CNR improvement from the $2 \mathrm{Tx} / 1 \mathrm{Rx}$ CDD. The simulation is carried out assuming 2-antenna transmitters $(2 T x)$ and 2-antenna receivers (2Rx). For 2Tx with transmitter diversity, the signals are transmitted using CDD. For $2 \mathrm{Rx}$ with receiver diversity, the signals are combined using MRC (Maximal Ratio Combining). The single-antenna $(1 \mathrm{Tx} / 1 \mathrm{Rx})$ system in which there is no CDD is simulated for reference. Second, the CNR gain of CDD in 2Tx/1Rx system is applied into the basic network planning tool to calculate the network coverage improvement in terms of transmitter power, transmitter antenna height and CNR ratio. As for the physical layer, the simulation of CDD diversity over the multipath channel model is based on three different radio environments defined as Typical Urban (TU), Rural Area (RA) and Indoor-B in UHF band. The power delay profiles for the TU and RA are specified by COST207 [15], and Indoor-B is specified by ITU-R [13]. Tables I, II and III give the values of the tap delays and the associated mean powers of TU, RA and Indoor-B. To enable good mobile reception, the DVB-H system is configured as follows: $4 \mathrm{~K}$ mode where the number of subcarriers used
TABLE I

Power Delay Profile of TyPical URban (TU)

\begin{tabular}{|l|l|l|}
\hline \hline \multicolumn{1}{|c|}{ TAPS } & \multicolumn{1}{|c|}{ Relative delay (us) } & Fading/dB \\
\hline 0 & 0 & -4 \\
\hline 1 & 0.2 & -3 \\
\hline 2 & 0.4 & 0 \\
\hline 3 & 0.6 & -2 \\
\hline 4 & 0.8 & -3 \\
\hline 5 & 1.2 & -5 \\
\hline 6 & 1.4 & -7 \\
\hline 7 & 1.8 & -5 \\
\hline 8 & 2.4 & -6 \\
\hline 9 & 3.0 & -9 \\
\hline 10 & 3.2 & -11 \\
\hline 11 & 5.0 & -10 \\
\hline
\end{tabular}

TABLE II

Power Delay Profile of Rural ARea (RA)

\begin{tabular}{|l|l|l|}
\hline \hline \multicolumn{1}{|c|}{ TAPS } & Relative delay (us) & Fading/dB \\
\hline 0 & 0 & 0 \\
\hline 1 & 0.1 & -4 \\
\hline 2 & 0.2 & -8 \\
\hline 3 & 0.3 & -12 \\
\hline 4 & 0.4 & -16 \\
\hline 5 & 0.5 & -20 \\
\hline
\end{tabular}

TABLE III

POWER DELAY PROFILE OF INDOOR-B

\begin{tabular}{|l|l|l|}
\hline \hline \multicolumn{1}{|c|}{ TAPS } & \multicolumn{1}{|c|}{ Relative delay (us) } & Fading/dB \\
\hline 0 & 0 & 0 \\
\hline 1 & 0.1 & -3.6 \\
\hline 2 & 0.2 & -7.2 \\
\hline 3 & 0.3 & -10.8 \\
\hline 4 & 0.5 & -18 \\
\hline 5 & 0.7 & -25.2 \\
\hline
\end{tabular}

is 4096 in a bandwidth of $8 \mathrm{MHz}$, QPSK, code rate 1/2, and guard interval $1 / 4$. The carrier frequency used is $900 \mathrm{MHz}$ and the mobile velocity 10 meter/second in which the equivalent Doppler frequency is $30 \mathrm{~Hz}$. These configurations are applied to all simulations carried out in this paper.

Second, for the statistical network planning simulation, a studied geographical area is divided into groups of pixels. Different geographical areas are simulated according to ITU R-P 1546 [13], i.e. suburban and urban. ITU R-P1546 is an ITU recommendation for field strength prediction. It can be used without taking the actual terrain into account. The curves in ITU R-P1546-1 represent the field strength in the VHF and UHF frequency bands as a function of different parameters. The model is based on a vast number of field strength measurements made over many years and condensed into curves so that the field strength at a chosen distance from a transmitter can be calculated. The propagation curve for a given value of field strength represents the field strength exceeding that value in $50 \%$ of the locations typically within an area of $200 \mathrm{~m}$ by $200 \mathrm{~m}$ for $1 \%, 10 \%$ or $50 \%$ of the time; The propagation loss curves for $50 \%$ of the time were used to calculate of useful signal and for $1 \%$ of the time were used to calculate the inference signal. One pixel represents one grid area. A pixel can be taken to be 


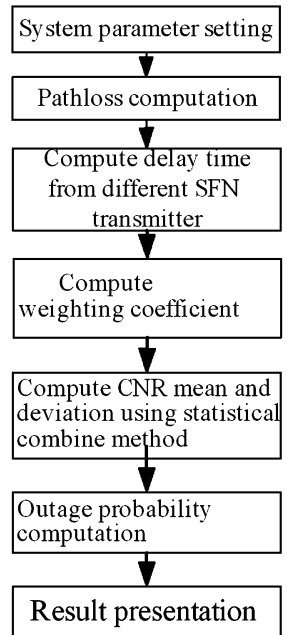

Fig. 2. Flow chart of the coverage simulation for one pixel.

$100 \mathrm{~m} \times 100 \mathrm{~m}, 200 \mathrm{~m} \times 200 \mathrm{~m}$ or an even bigger area of the studied region depending on the type of area studied. The studied area is decomposed into such pixels. The coverage of such a pixel is defined as "good" if at least $95 \%$ of receiving locations at the edge of the area are covered for portable reception and $99 \%$ of these receiving locations within it are covered for mobile reception. As for the "acceptable" locations, at least $70 \%$ of locations at the edge of the area are covered for portable reception and $90 \%$ of these receiving locations within it are covered for mobile reception [16].

The physical simulations characterize of this paper the sensitivity of the receiver in a fast fading environment. Physical layer simulations should not take account of the slow fading effect. Reception at the physical layer can definitely be used in network planning as this method is used by the DVB-T/H standards. For the studied area it is required that $90 \%$ of pixels are covered Fig. 2 gives the flowchart for the computation of the outage probability for one pixel only. For the other pixels in the studied area, the computation process is then repeated.

Since the receiver has different design characteristics for different manufacturers, a general receiver model is difficult to obtain. Rather than computing the network performance on one receiver design, in this paper, a range of CNR thresholds are used in the simulations based on the simulation results in the DVB-H standard [16]. In this paper, the CNR is computed based on the maximum CNR the receiver can obtain in the presence of the contributed and interference signals. Also, it is assumed in this paper that all the transmitters have the same transmitting power and all the transmit antennas are omni directional with the same height. The first signal to arrive at one receiving location is also the strongest one without a terrain model. In this case, the maximum CNR can be obtained when the start time of FFT window is aligned with the first signal received. The algorithm used to calculate the coverage radius can be found in [17]. The network simulation parameters are based on Table IV.

\section{A. Simulation Results for $C D D$}

Figs. 3, 4 and 5 show the BER (Bit Error Rate) performance for the TU, RA and ID-B radio environments with CDD transmit
TABLE IV

SIMULATION PARAMETERS

\begin{tabular}{|l|l|}
\hline Parameters & Value \\
\hline Transmitter frequency $(\mathrm{MHz})$ & 900 \\
\hline Transmitter power $(\mathrm{dBW})$ & $10 \sim 45$ \\
\hline Transmitter antenna height $(\mathrm{m})$ & $20 \sim 300$ \\
\hline Receiver height $(\mathrm{m})$ & 1.5 \\
\hline Transmitter antenna pattern & Omni \\
\hline Symbol time $(\mu \mathrm{s})$ & 448 \\
\hline Guard interval time $(\mu \mathrm{s})$ & $\begin{array}{l}112(1 / 4 \text { of symbol } \\
\text { time })\end{array}$ \\
\hline Receiver noise level $(\mathrm{dBW})$ & $\begin{array}{l}-129(\text { with a receiver } \\
\text { noise factor }=6 \mathrm{~dB})\end{array}$ \\
\hline Shadowing deviation $(\mathrm{dB})$ & 6 \\
\hline CNR threshold $(\mathrm{dB})$ & $9.7 ; 11 ; 14 ; 17$ \\
\hline & \\
\hline
\end{tabular}

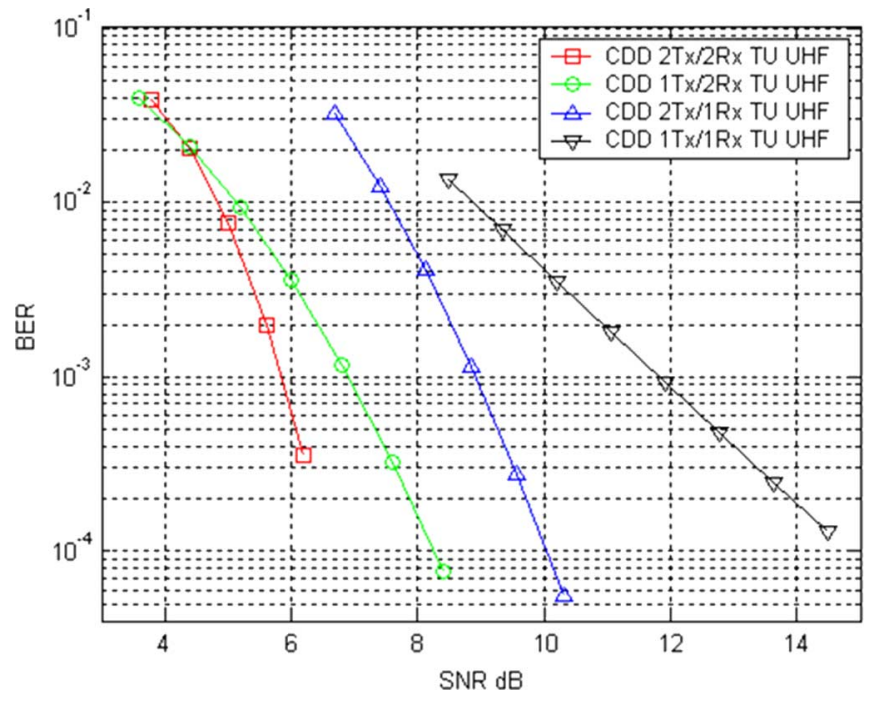

Fig. 3. Performance of CDD DVB-H in uncorrelated TU.

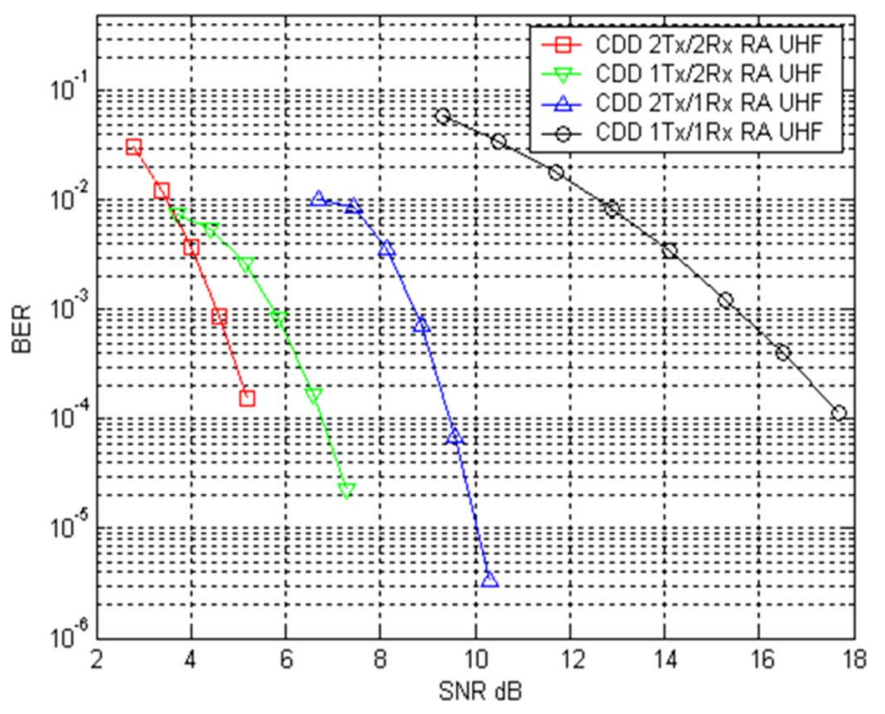

Fig. 4. Performance of CDD DVB-H in uncorrelated RA.

diversity applied to the DVB-H system in $4 \mathrm{~K}$ mode with QPSK modulation and code rate $1 / 2$. The simulations are carried out with assuming 2-antenna transmitters (2Tx) and 2-antenna receivers $(2 \mathrm{Rx})$. For $2 \mathrm{Rx}$ with receive diversity, the signals are 


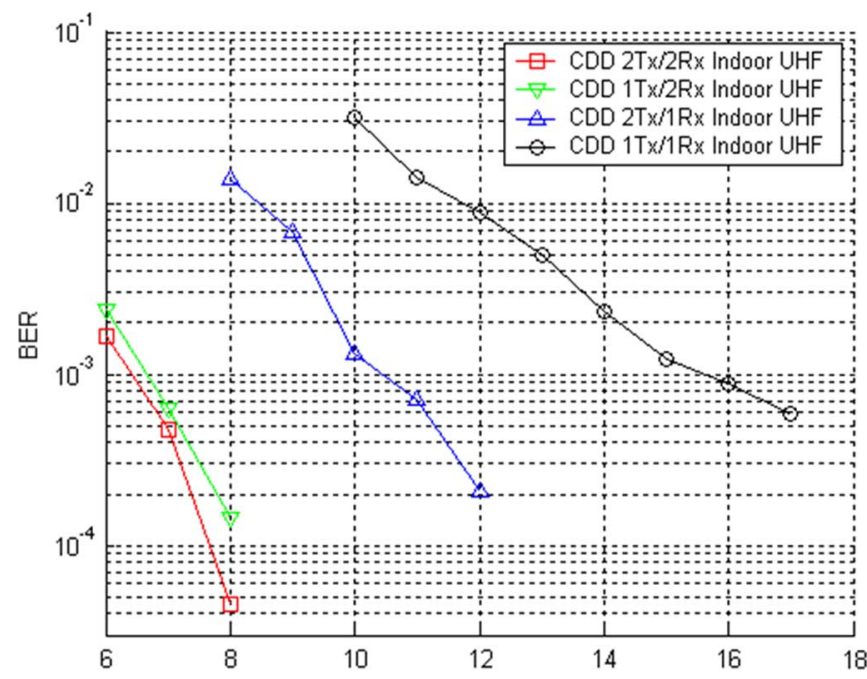

Fig. 5. Performance of CDD DVB-H in uncorrelated Indoor-B.

combined using MRC. The single-antenna (1Tx/1Rx) system in which there is no spatial diversity is simulated for reference.

CDD transmit diversity exploits the scattered signal propagation paths and with the receiver diversity the subcarriers that are deep faded at one receiver antenna may have good channel properties at the other receiver antenna. The cyclic delay for the simulation is about $1 \mu \mathrm{s}$. The BER is obtained after Viterbi decoder. The BER threshold for comparison diversity gain is $2 \times 10-4$ criterion as in DVB-T. It has been assumed that a Quasi Error Free condition of post-Viterbi BER at $2 \times 10-4$ is applicable for the situations modeled and diversity gains have been calculated at this level. The standard DVB-H physical receiver is regarded as the receiver for the simulation. From these figures, when comparing $2 \mathrm{Tx} / 1 \mathrm{Rx}$ to $1 \mathrm{Tx} / 1 \mathrm{Rx}$, it is observed that the diversity gain achieved in RA is the highest at about $7.3 \mathrm{~dB}$, followed by Indoor-B $(6 \mathrm{~dB})$ and $\mathrm{TU}(4.5 \mathrm{~dB})$. Note that the $\mathrm{TU}$ is frequency-selective and the channel does not undergo deep fading due to the high maximum channel delay of 5 us. The impairment caused by this delay is inherently mitigated by the OFDM system itself; thus CDD makes little improvement. On the other hand, the RA channel is non-frequency-selective (flat fading) with a severe deep fading because of the rather short maximum channel delay of $500 \mathrm{~ns}$; in this case, CDD increases the frequency-selectivity which explains the higher diversity gain compared to TU. It is noticed that CDD works well especially when the channel is undergoing deep fading which is usually caused by a shorter maximum channel delay e.g. RA and Indoor-B.

\section{B. Simulation Results for CDD Coverage Improvement}

If different SFNs use different frequencies to compose a wide area network, then interference coming from the other SFNs that use the same frequency will impair the reception quality in each SFN that uses that frequency. If the reuse factor is the number of frequencies reused in the wide area network and SFN size is the number of transmitters in the SFN, then Fig. 6 shows a single SFN of size 3 and reuse factor 7 in a two tier layout. For this layout 18 SFNs other than the studied SFN need to be considered for co-channel outer interference. This two-ring

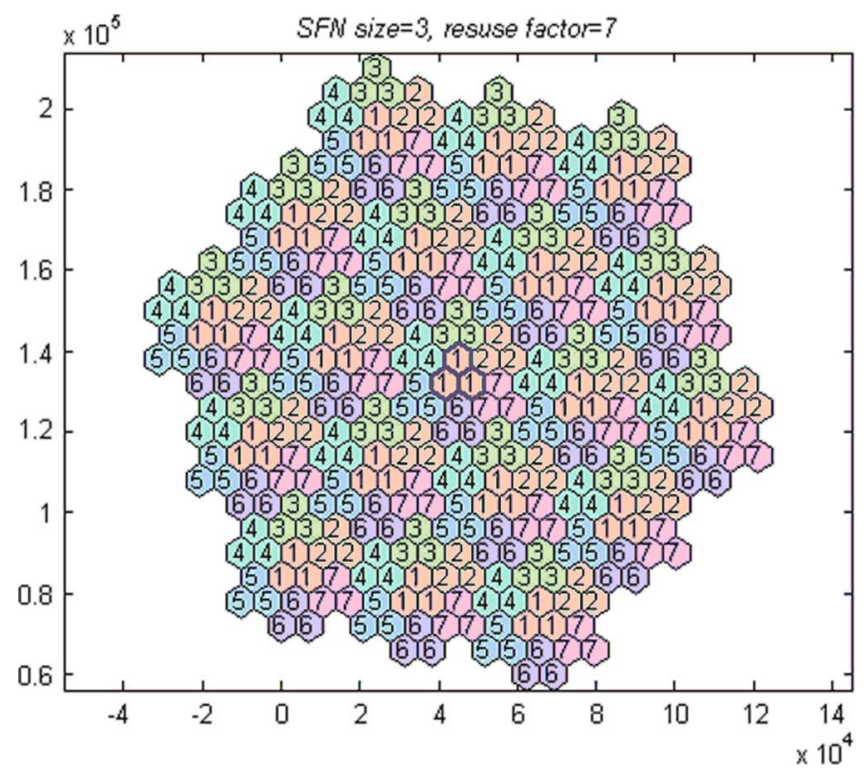

Fig. 6. Two tiers SFN networks SFN size $=3$ and Reuse factor $=7$ (different number represents different frequencies).

topology was taken as an example in [19] to study the outage probability in the central SFN.

The two tier layout of Fig. 6 is used in this paper to evaluate the performance of the SFN size 3 with different frequency reuse factors. The signal frequency was taken to be $900 \mathrm{MHz}$ in the UHF band. The transmit power and antenna height are in the ranges listed in Table IV. When all transmitters have the same transmitter power, all the antenna heights are the same, and all the antennas are omni directional, the first arrived signal at one receiving location with no terrain model is the strongest one. In this case, the maximum CNR can be obtained when the start time of the FFT window is aligned with the first received signal.

The concepts of location percentage and location correction are used in [16]. For the location percentage requirement for mobile reception, $13 \mathrm{~dB}$ of the location correction for $99 \%$ coverage target is taken. The location percentage requirement means the different percentage for the coverage target including 95\% for "good" area and 70\% for the "acceptable" area. The location correction is required to compensate for the rapid failure rate of digital TV signals defined in [16]. The mean value of $11 \mathrm{~dB}$ is taken for the UHF band building penetration loss [16]. The transmit antenna pattern is omni-direction and the antenna height is same for all the transmitters in a network topology. According to the diversity gain of TU, RA and Indoor-B channels in Fig. 3, 4, 5, the coverage improvement for the target BER 2x10-4 is shown in Fig. 7, 8, 9.

As for the TU channels, based on the Section IV-A results, the CNR threshold with transmitter diversity in the coverage planning is $9.7 \mathrm{~dB}$ and the CNR threshold without transmitter diversity in the coverage planning is $14 \mathrm{~dB}$. The coverage improvement for the DVB-H networks under different transmit powers and different antenna heights for a $90 \%$ coverage requirement in the studied area is shown in Fig. 7. The reuse factor is 7 in Fig. 7, when the transmit antenna height is $150 \mathrm{~m}$ and the coverage distance is $5000 \mathrm{~m}$, the transmit power with CDD is about $36 \mathrm{dBW}$ ("B" in Fig. 7) and the transmit power of the network 


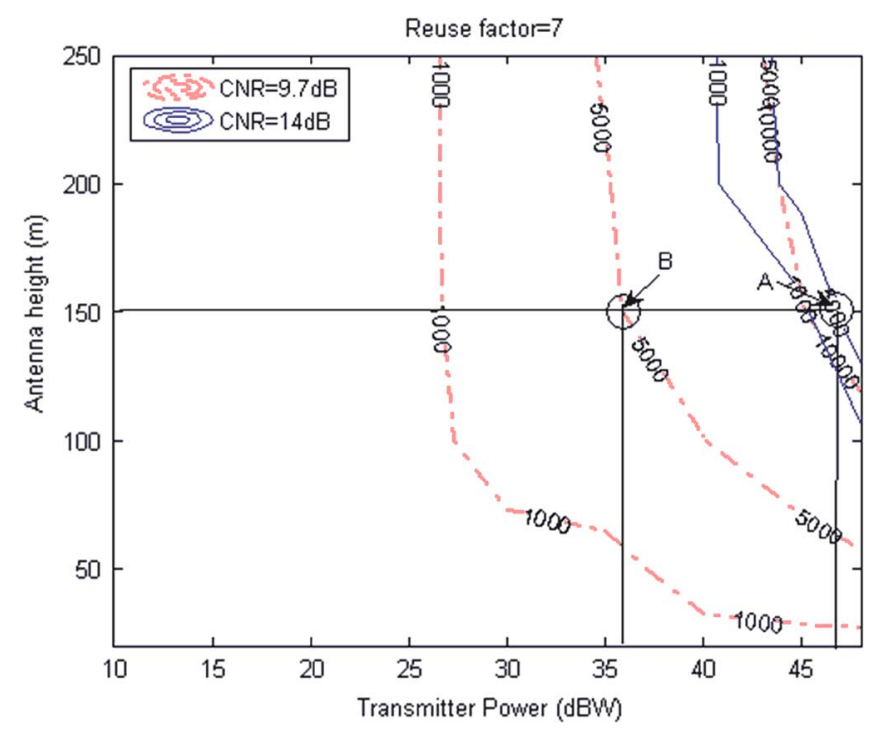

Fig. 7. Coverage improvement for Reuse factor $=7$ in TU channel.

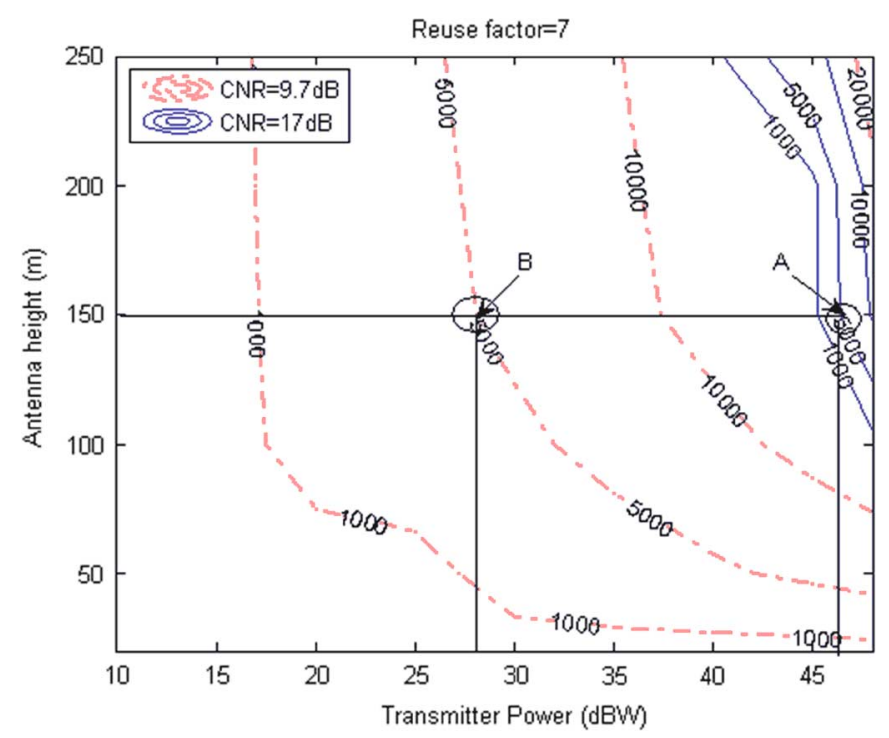

Fig. 8. Coverage improvement for Reuse factor $=7$ in RA channel.

without CDD is about 47 dBW ("A" in Fig. 7). There is about $11 \mathrm{dBW}$ transmit power gain for the same $5000 \mathrm{~m}$ coverage radius.

As for the RA channels, based on the Section IV-A results, the CNR threshold with transmitter diversity in the coverage planning is $9.7 \mathrm{~dB}$ and the CNR threshold without transmitter diversity in the coverage planning is $17 \mathrm{~dB}$. The reuse factor is 7 in Fig. 8, when the transmit antenna height is $150 \mathrm{~m}$ and the coverage distance is $5000 \mathrm{~m}$, the transmit power with CDD is about $28 \mathrm{dBW}$ ("B" in Fig. 8) and the transmit power of the network without CDD is about $47 \mathrm{dBW}$ ("A" in Fig. 8). There is about a $19 \mathrm{~dB}$ transmit power gain for the same $5000 \mathrm{~m}$ coverage radius.

As for the Indoor-B channels, based on the Section IV-A results, CDD can get a $6 \mathrm{~dB}$ diversity gain in CNR for the DVB-H network planning. The reuse factor is 7 in Fig. 9, when the transmit antenna height is $150 \mathrm{~m}$ and the coverage distance is

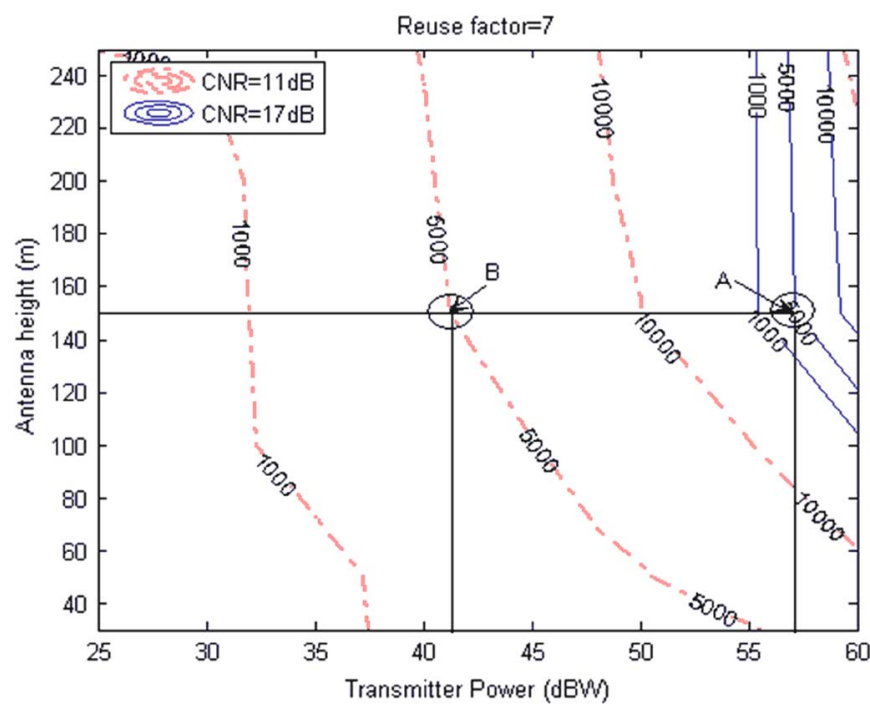

Fig. 9. Coverage improvement for Reuse factor $=7$ in Indoor-B channel.

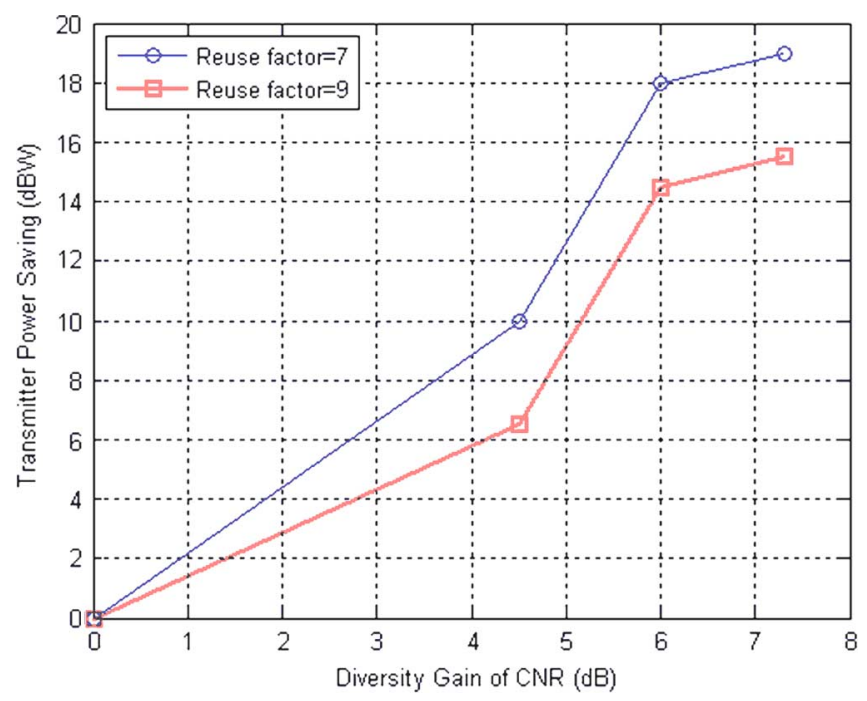

Fig. 10. Transmitter power saving vs. diversity gain of CNR with different reuse factors in RA channel.

$5000 \mathrm{~m}$, the transmit power with CDD is about $41 \mathrm{dBW}$ ("B" in Fig. 9) and the transmit power of the network without CDD is about $57 \mathrm{dBW}$ ("A" in Fig. 9). There is about a $16 \mathrm{~dB}$ transmit power gain for the same $5000 \mathrm{~m}$ coverage radius.

Fig. 7, 8, and 9, show CDD can deliver $11 \mathrm{~dB}, 19 \mathrm{~dB}$ and $16 \mathrm{~dB}$ transmitter power savings in TU, RA and indoor-B channels respectively with reuse factor 7 . The transmitter power saving rate varies with the reuse factor, antenna height and the diversity gain in CNR [20]. Therefore, for fixed antenna height, the transmitter power saving rate is shown in Fig. 10 in terms of reuse factor and diversity gain in RA channel. In the simulation, the different diversity gains in RA channel are regarded as the input parameters for the network planning tools.

Fig. 10 shows the transmitter power saving versus diversity gain in CNR for reuse factors 7 and 9 in RA channel. The transmitter height is $150 \mathrm{~m}$. From Fig. 10, it is seen that the transmitter power saving is improved with increasing diversity gain in CNR. Furthermore, there is a threshold for the transmitter 


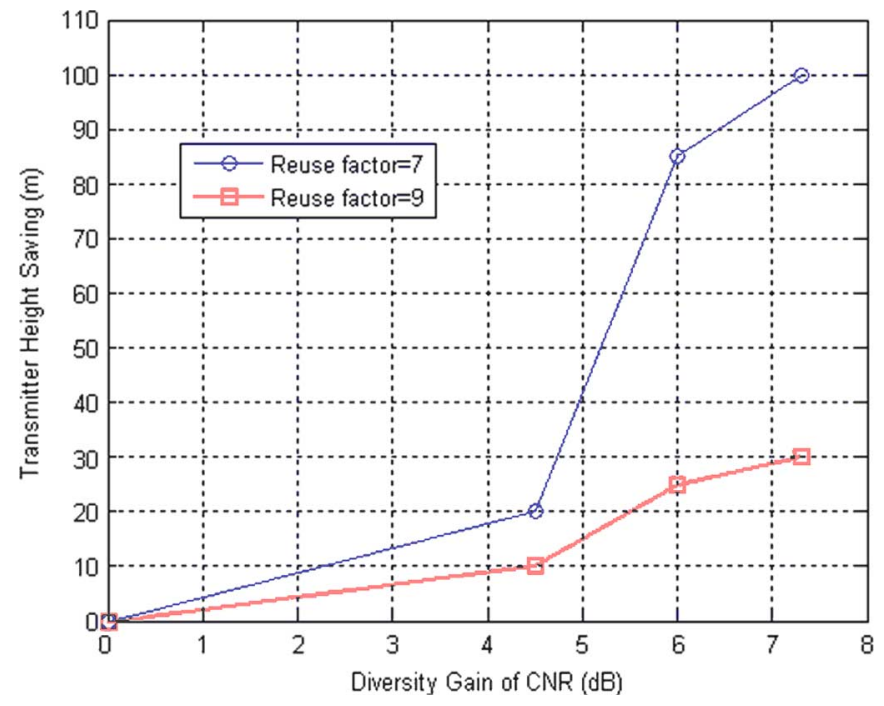

Fig. 11. Transmitter height saving vs. diversity gain of CNR with different reuse factors in RA channel.

power saving. For reuse factor 7 , when the diversity gain increases from $6 \mathrm{~dB}$ to $7.5 \mathrm{~dB}$, there is only a $1 \mathrm{dBW}$ improvement in the transmitter power saving. However, when the diversity gain increases from $4.5 \mathrm{~dB}$ to $6 \mathrm{~dB}$, there is a $8 \mathrm{dBW}$ improvement in the transmitter power saving. Moreover, the transmitter power saving rate also depends on the reuse factor of the network. For a diversity gain in CNR of $6 \mathrm{~dB}$, the transmitter power saving for reuse factor 9 is $13.5 \mathrm{dBW}$ and the transmitter power saving for reuse factor 7 is $18 \mathrm{dBW}$. Therefore, the power saving rate decreases as the reuse factor of the network increasing.

Fig. 11 shows the transmitter height saving versus diversity gain in CNR for reuse factors 7 and 9 in RA channel. The transmitter power is $48 \mathrm{dBW}$ and the coverage radius is $5000 \mathrm{~m}$. From Fig. 11, it can be seen that the transmitter height saving is improved with increasing diversity gain in CNR. When the diversity gain is $4.5 \mathrm{~dB}$, there is about a $20 \mathrm{~m}$ saving in the transmitter height to cover $5000 \mathrm{~m}$ for reuse factor 7 in RA channel. For reuse factor 9 , there is about a $10 \mathrm{~m}$ saving. When the diversity gain is $7.3 \mathrm{~dB}$ the transmitter height can be reduced by $100 \mathrm{~m}$ for reuse factor 7 and by $30 \mathrm{~m}$ for reuse factor 9 . Thus the saving in transmitter height depends on the reuse factor of the network. For a diversity gain in CNR of $6 \mathrm{~dB}$, the transmitter height saving for reuse factor 9 is $25 \mathrm{~m}$ and the transmitter height saving for reuse factor 7 is $85 \mathrm{~m}$.

Fig. 12 shows the coverage improvement versus diversity gain in CNR for reuse factors 7 and 9 in RA channel. The transmitter power is $48 \mathrm{dBW}$ and the transmitter height is $150 \mathrm{~m}$. From Fig. 12 it can be seen that the network coverage is improved with increasing diversity gain in CNR. When the diversity gain is $4.5 \mathrm{~dB}$, the coverage improvement is about $2500 \mathrm{~m}$ for reuse factor 7 and about $1000 \mathrm{~m}$ for reuse factor 9. When the diversity gain is $7.3 \mathrm{~dB}$, the coverage improvement is about $8000 \mathrm{~m}$ for reuse factor 7 and about $5000 \mathrm{~m}$ for reuse factor 9 . Thus the network coverage improvement depends on the reuse factor of the network for a given transmitter power and height.

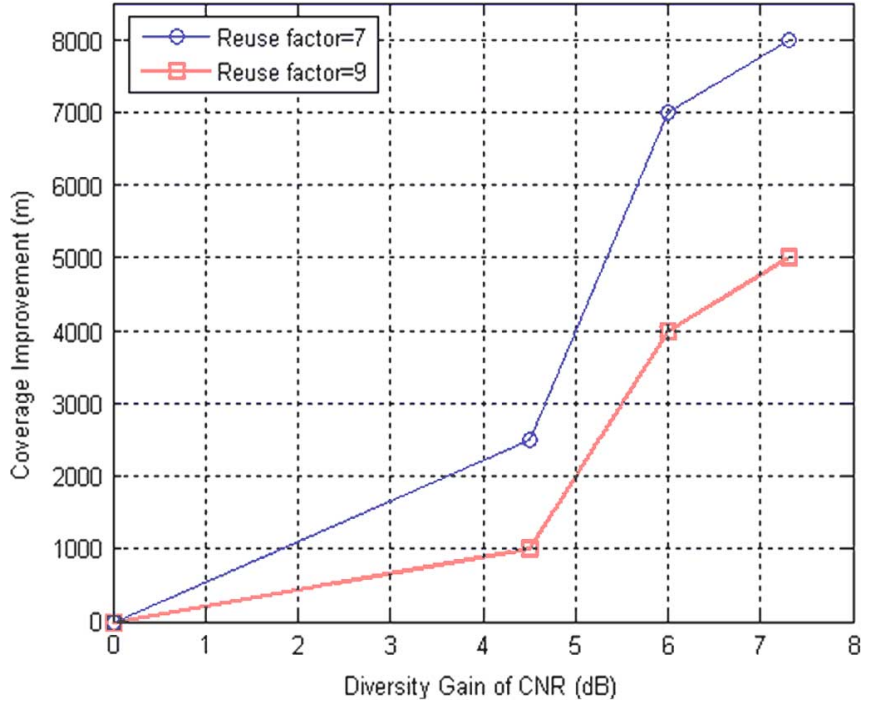

Fig. 12. Coverage improvement vs. diversity gain of CNR with different reuse factors in RA channel.

\section{CONCLUSION}

This paper has presented an investigation of DVB-H network coverage with the application of CDD transmit diversity. The channel model and the simulations follow a statistical approach for the sake of simplicity. The simulation results suggest that CDD can deliver improvements of about $7.3 \mathrm{~dB}, 6 \mathrm{~dB}$ and $4.3 \mathrm{~dB}$ in CNR threshold in RA, Indoor-B and TU environments, respectively, with 2 transmitter antennas and 1 receiver antenna compared with the standard 1Tx/1Rx system and thus assists DVB-H SFN network planning. In addition, CDD reduces the network costs in terms of the transmit power, antenna height and frequency reuse factor and improves the DVB-H cellular network coverage. There are about $11 \mathrm{dBW}, 16 \mathrm{dBW}$ and $19 \mathrm{dBW}$ gains in transmit power for $5000 \mathrm{~m}$ coverage radius for reuse factor 7 with transmit antenna height at $150 \mathrm{~m}$ for CDD with 2Tx/1Rx DVB-H systems in TU, Indoor-B, and RA channels, respectively. Furthermore, the gain in transmitter power increases by increasing the CDD diversity gain in CNR. There is a threshold for the gain in transmitter power in terms of the CDD diversity gain in CNR. The transmitter height can be decreased as the diversity gain in CNR increases. For given transmitter height and transmitter power level, the greater the diversity gains the greater the network coverage improvement. Finally, the gain in transmitter power decreases by the increasing the frequency reuse factor. As perspectives to this work, it is envisaged to use other channel models including site-specific deterministic propagation tools to refine the analysis on special cases. Furthermore, later studies will include the effect of Reed Solomon coding and define reception thresholds in terms of uncorrectable Reed-Solomon errors.

\section{ACKNOWLEDGMENT}

The authors would like to express their special thanks to all the PLUTO project partners for their valuable contributions to 
this research. The reviewers are thanked for comments that significantly improved the readability of the paper.

\section{REFERENCES}

[1] European Telecommunications Standard Institute ETSI York, "Digital Video Broadcasting (DVB); DVB-H Implementation Guidelines," TR 102377 V1.1.1, Feb. 2005.

[2] A. Mattsson, "Single frequency networks in DTV," IEEE Trans. Broadcasting, vol. 51, no. 4, pp. 413-422, Dec. 2005.

[3] J.-T. Wang, J. Song, J. Wang, C.-Y. Pan, Z.-X. Yang, and L. Yang, "A general SFN structure with transmit diversity for TDS-OFDM system," IEEE Trans. Broadcasting, vol. 52, no. 2, pp. 245-251, June 2006.

[4] M. Kornfeld and G. May, "DVB-H and IP Datacast-broadcast to handheld devices," IEEE Trans. Broadcasting, vol. 53, no. 1, pp. 161-170, March 2007.

[5] European Telecommunications Standard Institute ETSI York: Digital Video Broadcasting (DVB), "Framing Structure, Channel Coding and Modulation for Digital Terrestrial Television," EN 300744 V1.2.1., July 1999.

[6] A. Dammann and S. Kaiser, "Standard conformable antenna diversity techniques for OFDM systems and its application to the DVB-T system," in IEEE Globecom, Nov. 2001, pp. 3100-3105.

[7] Y. Zhang, J. Cosmas, M. Bard, and Y. H. Song, "Diversity gain for DVB-H by using transmitter/receiver cyclic delay diversity," IEEE Trans. Broadcasting, vol. 52, no. 4, pp. 464-474, Dec. 2006.

[8] Y. Zhang, J. Cosmas, M. Bard, and Y. H. Song, "Future transmitter/receiver diversity schemes in broadcast wireless networks," IEEE Communications Mag, vol. 44, no. 10, pp. 120-127, Oct. 2006.

[9] Y. Wu, X. B. Wang, R. Citta, L. Benoit, L. Sebastien, and C. Bernard, "An ATSC DTV receiver with improved robustness to multipath and distributed transmission environments," IEEE Trans. Broadcasting, vol. 50, no. 1, pp. 32-41, March 2004.

[10] Y. Wu, S. Hirakawa, U. H. Reimes, and J. Whitaker, "Overview of digital television development worldwide," Proceedings of the IEEE, vol. 94, no. 1, pp. 8-21, Jan. 2006.

[11] H. Xiong, J. Sun, S. Yu, J. Zhou, and C. Chen, "Rate control for realtime video network transmission on end-to-end rate-distortion and application-oriented QoS," IEEE Trans. Broadcasting, vol. 51, no. 1, pp. 122-132, March 2005.

[12] Y. Yeh and S. Schwartz, "Outage probability in Mobile Telephony due to multiple Log-Normal interferes," IEEE Trans. Communications, vol. COM-32, no. 4, Apr. 1984.

[13] "ITU-R Recommendation P.1546: Method for Point-to-Area Predictions for Terrestrial Services in the Frequency Range $30 \mathrm{MHz}$ to 3000 MHz," [Online]. Available: http://www.itu.int/itudoc/itu-r/rec/p/index. html

[14] N. C. Beaulieu, A. A. Abu-Dayya, and P. J. McLane, "Comparison of methods of computing lognormal sum distributions and outages for digital wireless applications," in IEEE International Conference on $\mathrm{Hu}$ manity Through Communications, May 1-5, 1994.

[15] COST 207, "Digital land mobile radio communications," Office for Official Publications of the European Communities, Abschlussbericht, Lemburg, 1989.

[16] "European Telecommunications Standard Institute ETSI: Digital Video Broadcasting (DVB)- DVB-H Implementation Guidelines," ETSI TR 102377 V1.2.1; 2005-11

[17] C. Zhang, T. J. Owens, and Y.-H. Song, "On the performance of densified single frequency networks for DVB-H," International Journal of Mobile Network Design and Innovation, vol. 1, no. 3/4, pp. 224-233.

[18] R. Brugger and D. Hemingway, "OFDM receivers: Impact on coverage of inter-symbol interference and FFT window positioning," EBU Technical Review, July 2003.

[19] R. Rebhan and J. Zander, "On the outage probability in single frequency networks for digital broadcasting," IEEE Trans. Broadcasting, vol. 39, no. 4, pp. 395-401, Dec. 1993.

[20] Y. Zhang, J. Cosmas, K.-K. Loo, M. Bard, and R. Bari, "Analysis of cyclic delay diversity on DVB-H systems over spatially correlated channel," IEEE Trans. Broadcasting, vol. 53, no. 1, pp. 247-255, March 2007.

\section{[21] “IST Project PLUTO,” [Online]. Available: http://www.ist-pluto.org}

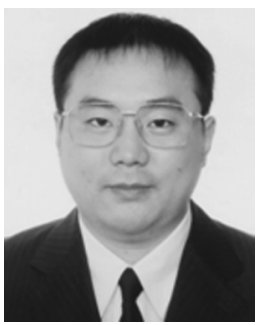

Yue Zhang (M'06) Studied Telecommunications Engineering in Beijing University of Posts and Telecommunications (BUPT) and received B.Eng and M.Eng degrees in 2001 and 2004 respectively. He obtained $\mathrm{PhD}$ degree in electronics engineering at Brunel University in 2008. He had worked for Brunel University as research assistant over two years for IST FP6 PLUTO project, which is to investigate and measure the MIMO effects over DVB-T/H networks. He also designed and implemented the low cost On-Channel repeater in DVB-T/H networks with digital echo cancellation in DSP and FPGA. His research interests are signal processing, wireless communications systems, MIMO-OFDM systems, radio propagation model and multimedia and wireless networks. He currently works in Anritsu Company as signal processing design engineer. He has published over 10 papers in refereed conference proceedings and journals. He also serves as a reviewer for IEEE TRANS ON BROADCASTING, wireless communication, circuits and systems I (CAS I) and guest editor for international journal of digital multimedia broadcasting.

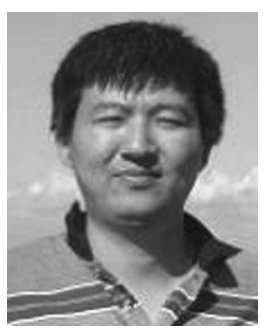

Chunhui Zhang obtained a B.Eng honors degree in Electronic Engineering at Tsinghua University in 1999 and a PhD in communication at Brunel University in 2006. He is a system designer in Ericsson. His research interests are concerned with the network planning for DTV/DAB networks.

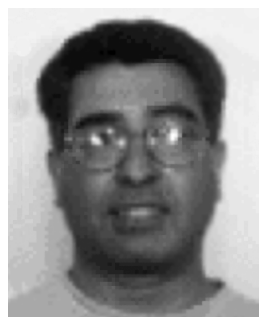

John Cosmas (M'86) obtained a B.Eng honors degree in Electronic Engineering at Liverpool University in 1978 and a PhD in Image Processing and Pattern Recognition at Imperial College, University of London in 1987. He is a Professor of Multimedia Systems and became a Member (M) of IEEE in 1987 and a Member of IEE in 1977. His research interests are concerned with the design, delivery and management of new TV and telecommunications services and networks, multimedia content and databases, and video/image processing. He has contributed towards eight EEC research projects and has published over 80 papers in refereed conference proceedings and journals. He leads the Networks and Multimedia Communications Centre within the School of Engineering and Design at Brunel University.

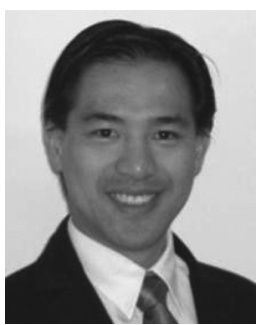

Kok-Keong Loo (M'01) a.k.a. Jonathan Loo received his MSc degree (Distinction) in Electronics at University of Hertfordshire, UK in 1998 and $\mathrm{PhD}$ degree in Electronics and Communication at the same university in 2003. After completing his PhD, he works as a lecturer in multimedia communications at Brunel University, UK. He is also a course director for MSc Digital Signal Processing. Besides that, he currently serves as principle investigator for a joint project between Brunel University and British Broadcasting Corp (BBC) on the Dirac video codec research and development. He also serves as co-investigator for the IST-FP6 PLUTO project. His current research interests include visual media processing and transmission, digital/wireless signal processing, software defined radio, and digital video broadcasting and networks. 


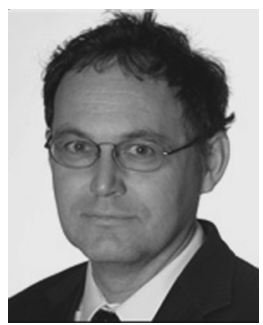

refereed papers in journals

Thomas Owens obtained his $\mathrm{PhD}$ in Electrical and Electronic Engineering from Strathclyde University in 1986. In 1987 he joined as a lecturer the Department of Electronic and Electrical Engineering, Brunel University, which was eventually absorbed into the School of Engineering and Design in 2004 in which he is Senior Lecturer Communications. $\mathrm{He}$ was the project coordinator of the IST FP5 project CONFLUENT, the IST FP6 Integrated Project INSTINCT, and the FP6 Specific Support Action PARTAKE. He is the author of more than forty

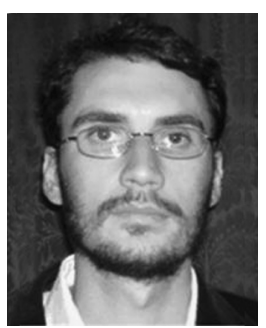

Raffaele Di Bari received the B.S. and M.S. in telecommunications engineering from Pisa University, Pisa, Italy, in 2003 and 2005, respectively. $\mathrm{He}$ is currently working toward the Ph.D. degree in the Department of Electrical and Computer Engineering, University of Brunel, Uxbridge. His current research interests are in the area of Digital Video Broadcasting, MIMO-OFDM systems and Radio Channel measurements. Since 2006, he also is a participant of PLUTO project.

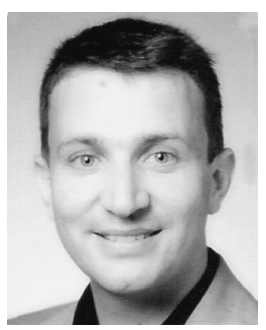

Yves Lostanlen (S'98-M'01) obtained a Diplomarbeit at Friedrich Alexander Universitaet, Erlangen, Germany and received the Dipl.-Ing (M.S.E.E) in 1996 from National Institute for Applied Sciences (INSA) in Rennes. After three years of research at University College London and INSA Rennes he accomplished a European Dr.-Ing. (Ph.D.E.E) with honors in 2000.

He is currently Director of the Radio R\&D Department at Siradel, Rennes, France where he manages a team of radio planning consultants, software devel- opers, software support, researchers, radio R\&D engineers carrying out research into RF propagation applied to Radio communication Systems and Digital TV $\mathrm{He}$ is also responsible for managing the Scientific Communication and assisting with Scientific Marketing for the company.

Dr. Lostanlen is a telecommunications expert and manager with over ten years experience and involvement with government, operators and manufacturers. He acts as a consultant for public, military and private organizations including major wireless industry players. He is currently Task and Work Package Leader in the European IST-PLUTO, ICT-WHERE, ICT-UCELLS projects.

Dr. Lostanlen regularly holds lectures, tutorials, seminars, workshops and trainings in industrial and academic institutions. He is engaged in several leading industry and academic bodies including SEE, IEEE, COST 273 and COST2100. From 2001 to 2008 he was appointed member of the French committee IEEE Antennas and Propagation.

Yves Lostanlen has written more than 50 papers for international conferences, periodicals, book chapters and has been session chairman and member of scientific committees at several international conferences. He received a "Young Scientist Award" for two papers at the EuroEM 2000 conference.

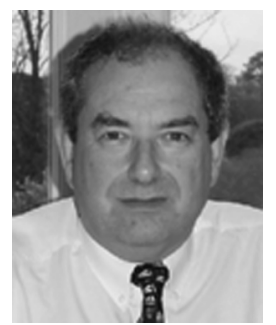

Maurice Bard graduated from Imperial College in 1976 with a BSc (Hon) in Materials Science and worked initially on Travelling Wave Tube design, electronics systems and software. Maurice has succeeded in a number of engineering, sales and marketing roles during a 20 year career at Nortel Networks. Whilst there he founded and managed a business providing GPS Simulators to a world market before moving on to establish a new Fixed Wireless product line which deployed 1 million lines around The World. He left to join PipingHot Networks in 2000; a wireless start-up which is now established as an international provider of Non-Line of Site radio links using similar principles to those proposed here. More recently Maurice has been working as an independent consultant in the wireless, broadcast and GPS industries. 